\title{
Fuzzy Control Model and Simulation for Nonlinear Supply Chain System with Lead Times
}

\author{
Songtao Zhang, ${ }^{1}$ Yanting Hou, ${ }^{2}$ Siqi Zhang, ${ }^{3}$ and Min Zhang $^{4}$ \\ ${ }^{1}$ School of Logistics, Linyi University, Linyi 276005, China \\ ${ }^{2}$ School of Economics and Management, Tongji University, Shanghai 200092, China \\ ${ }^{3}$ Faculty of Business and Economics, The University of Melbourne, Melbourne, VIC 3010, Australia \\ ${ }^{4}$ Library, Linyi University, Linyi 276005, China
}

Correspondence should be addressed to Songtao Zhang; zst0626@163.com

Received 18 February 2017; Revised 4 June 2017; Accepted 13 August 2017; Published 14 September 2017

Academic Editor: Pietro De Lellis

Copyright (c) 2017 Songtao Zhang et al. This is an open access article distributed under the Creative Commons Attribution License, which permits unrestricted use, distribution, and reproduction in any medium, provided the original work is properly cited.

\begin{abstract}
A new fuzzy robust control strategy for the nonlinear supply chain system in the presence of lead times is proposed. Based on Takagi-Sugeno fuzzy control system, the fuzzy control model of the nonlinear supply chain system with lead times is constructed. Additionally, we design a fuzzy robust $H_{\infty}$ control strategy taking the definition of maximal overlapped-rules group into consideration to restrain the impacts such as those caused by lead times, switching actions among submodels, and customers' stochastic demands. This control strategy can not only guarantee that the nonlinear supply chain system is robustly asymptotically stable but also realize soft switching among subsystems of the nonlinear supply chain to make the less fluctuation of the system variables by introducing the membership function of fuzzy system. The comparisons between the proposed fuzzy robust $H_{\infty}$ control strategy and the robust $H_{\infty}$ control strategy are finally illustrated through numerical simulations on a two-stage nonlinear supply chain with lead times.
\end{abstract}

\section{Introduction}

Over the recent years, a large number of companies realize the value-added importance of supply chain (SC) system and have cooperated as a part of it [1]. Efficient management of distribution, production, and supply in the SC has critical influence on business success [2]. However, SC system is more sensitive to the existence of lead time. Lead time, which is affected by the physical distance between the seller and the buyer, transportation mode, manufacturer's production capability, and technology in practice [3], can result in oscillation and instability of the SC system directly. Therefore, effectively restraining the impact of lead time on the SC system can be one of the major challenging issues to be resolved for the node companies in competition [4].

For the controllable lead time, Mahajan and Venugopal [5] studied the impacts of the reduction of lead time on the retailer and manufacturer's costs. For a two-stage SC consisting of a manufacturer and a retailer, Leng and Parlar
[6] investigated game-theoretic models of the reduction of lead time. According to the reduction of lead time caused by the added crashing cost, Li et al. [7] studied the coordination problem of a decentralized SC. Glock [8] proposed alternative approaches on the reduction of lead time and their impacts on the safety inventory and the expected total cost of the integrated inventory system. Further, a model of the divergent SC to study how to minimize the expected total cost and reduce lead times to find the optimal production, inventory, and routing decisions has been described by Jha and Shanker [9]. With the help of lead time variation control, Heydari [10] developed an incentive scheme to realize the service level coordination in a two-stage SC.

On the other hand, for the uncontrollable lead time, Garcia et al. [11] proposed an Internal Model Control (IMC) approach to control the production inventory in a SC with lead times. By utilizing the multimodel scheme, the IMC control approach can realize the online identification of lead times. In addition, $\mathrm{Xu}$ and Rong [12] utilized the minimum 
variance control theory to derive the order-up-to policy for the SC with time-varying lead time. Taleizadeh et al. [13] performed a particle swarm optimization to access the inventory problem of the chance-constraint joint single vendor-single buyer with changeable lead time. To the aim of restraining the bullwhip effect of uncertain SCs with vendor order placement lead time, a robust optimization strategy has been highlighted by Li and Liu [14]. Garcia et al. [15] incorporated an IMC scheme in production inventory control system of a complete SC to online identify lead times. Further, Han et al. [16] analyzed the approximate optimal inventory control problem of SC networks with lead time and proposed a suboptimal inventory replenishment strategy to effectively reduce bullwhip effect and improve the performance of SC networks system. Movahed and Zhang [17] formulated the inventory system of a single-product three-level multiperiod $\mathrm{SC}$ with uncertain demands and lead times as a robust mixedinteger linear program with minimized expected cost and total cost variation to determine the optimal s, $S$ values of the inventory parameters. Using the proportional control approach, Wang and Disney [18] mitigated the amplification of order and inventory fluctuations in a state-space SC model with stochastic lead time.

The SC system is only considered as a linear system whether with the controllable lead time or with the uncontrollable lead time. However, it is worth noting that the SC system is dynamic in the operational process due to the influences of the uncertain customers' demands and lead times. In this perspective, this leads to multiple possible strategies in manufacturing, delivering, and ordering products measured by the relation between upstream company's inventory level and downstream company's demand state. That is to say, the node companies of manufacturing or ordering can implement multiple strategies instead of one in different scenarios. In such a situation, a linear switching system with many modes can be performed instead of a unique SC model as well. Therefore, the SC system is nonlinear dynamic with piecewise linear characteristics. Nevertheless, the SC as a nonlinear dynamic system has been rarely addressed in the related literature.

Robust fuzzy control strategies for controlling nonlinear dynamic systems have been addressed broadly. For the nonlinear systems with uncertainties, Lee et al. [19] studied the fuzzy robust control problem for the continuous-time and discrete-time nonlinear systems with parametric uncertainties based on Takagi-Sugeno (T-S) fuzzy model and derived the sufficient conditions of robust stabilization in the sense of Lyapunov asymptotic stability; Yang and Zhao [20] presented a robust control approach for uncertain switched fuzzy system and designed a continuous state feedback controller to ensure the relevant closed-loop system is asymptotically stable for all allowable uncertainties. The nonlinear systems with time delay are also mentioned in a few literatures. Cui et al. [21] discussed the problem of robust $H_{\infty}$ control for a class of uncertain switched fuzzy time-delay systems described by T-S fuzzy model and derived a sufficient condition to guarantee the stability of the closed-loop systems. Further, Teng et al. [22] investigated the robust model predictive control of a class of nonlinear discrete system subjected to time delays and persistent disturbances. However, the robust control approaches in [19-22] cause higher conservative to guarantee the stability of the system by finding the common positive definite matrices.

In this paper, we will propose a fuzzy robust $H_{\infty}$ control strategy to restrain the impacts of lead times, switching actions among subsystems, and customers' stochastic demands on the nonlinear dynamic SC system. By utilizing the concept of maximal overlapped-rules group (MORG), the control strategy can be obtained from T-S fuzzy system associated with robust $H_{\infty}$ control method, which can guarantee the stability of the system if the local common positive definite matrices in each MORG can be found. Therefore, the proposed control strategy can (i) reduce the conservatism as compared with the existing control approaches; (ii) make SC system robustly asymptotically stable; and (iii) realize soft switching among subsystems of the nonlinear dynamic SC. We make some comparisons with the robust $H_{\infty}$ control strategy to demonstrate the effectiveness of our proposed strategy.

The rest of this paper is arranged as follows. The fuzzy model of the nonlinear dynamic SC system with lead times is formulated in Section 2. Then Section 3 proposes a new fuzzy robust $H_{\infty}$ control strategy. Finally, Section 4 provides an illustrative example of a two-stage nonlinear SC system with the production lead time and the ordering lead time to verify the advantage of the proposed control strategy. Our conclusions are presented in Section 5.

\section{Model Construction and Preliminaries}

2.1. Nonlinear Dynamic SC Fuzzy System. The formulation of a basic model of two-stage SC system with lead times (i.e., production lead time and ordering lead time) can be illustrated in Figure 1.

In Figure $1, x_{a}(k)$ and $x_{b}(k)$ denote manufacturer $(a)^{\prime}$ inventory level and retailer $(b)^{\prime}$ inventory level at period $k$, respectively, $u_{a}(k)$ and $u_{a}\left(k-\tau_{a}\right)$ denote the productions manufactured by manufacturer $(a)$ at period $k$ and with the production lead time $\tau_{a}$, respectively, $u_{b}(k)$ and $u_{b}\left(k-\tau_{b}\right)$ are the numbers of products ordered by retailer $(b)$ at period $k$ and with the ordering lead time $\tau_{b}$, respectively, and $w_{b}(k)$ is the customers' demands at period $k$.

Remark 1. Figure 1 can describe 4 kinds of SC systems: (1) when $a=1$ and $b=1$, Figure 1 denotes the chain-type SC system; (2) when $a=1$ and $b=2,3, \ldots, t$, Figure 1 denotes the distribution-type SC system; (3) when $a=2,3, \ldots, s$ and $b=1$, Figure 1 denotes retailers-centered multistage SC system, like supermarket. (4) when $a=1,2,3, \ldots, s$ and $b=1,2,3, \ldots, t$, Figure 1 denotes the SC network system.

The basic dynamic mathematical model of the SC system is presented as follows:

$$
\begin{aligned}
& x_{a}(k+1)=x_{a}(k)+u_{a}(k)+u_{a}\left(k-\tau_{a}\right)-u_{b}(k), \\
& x_{b}(k+1)=x_{b}(k)+u_{b}(k)+u_{b}\left(k-\tau_{b}\right)-w_{b}(k) .
\end{aligned}
$$




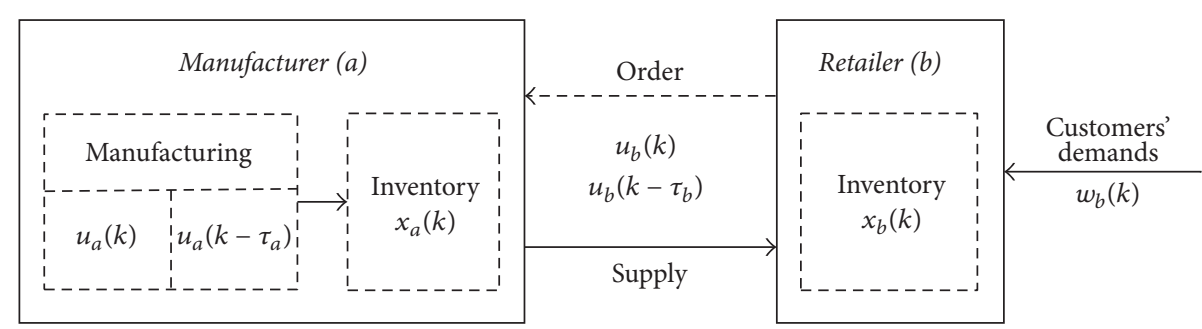

Figure 1: Basic dynamic model of two-stage SC system.

In the operational process of the SC system, the node companies will adopt different production or ordering strategies according to their own different inventory levels, which results in many different basic dynamic models, and the basic dynamic models can be called subsystems. Moreover, to reduce the total cost of this SC system, there exist switching actions among subsystems at different period $k$. Therefore, the dynamic SC system is a piecewise linear system, which can be also called a nonlinear system.

By utilizing the matrix theory and considering the total cost of the nonlinear SC system, the $i$ th subsystem of (1) can be described as follows:

$$
\begin{aligned}
\mathbf{x}(k+1)= & \mathbf{A}_{i} \mathbf{x}(k)+\mathbf{B}_{i} \mathbf{u}(k)+\sum_{e=1}^{n} \mathbf{B}_{i e} \mathbf{u}\left(k-\tau_{e}\right) \\
& +\mathbf{B}_{w i} \mathbf{w}(k), \\
\mathbf{z}(k)= & \mathbf{C}_{i} \mathbf{x}(k)+\mathbf{D}_{i} \mathbf{u}(k)+\sum_{e=1}^{n} \mathbf{D}_{i e} \mathbf{u}\left(k-\tau_{e}\right),
\end{aligned}
$$

where the subscript $i$ corresponds to the SC being in the $i$ th mode, $\mathbf{x}(k)=\left[\begin{array}{llll}x_{1}(k) & x_{2}(k) & \cdots & x_{n}(k)\end{array}\right]^{T}(n=s+t)$ is the inventory state variable, $\mathbf{u}(k)=\left[\begin{array}{llll}u_{1}(k) & u_{2}(k) & \cdots & u_{n}(k)\end{array}\right]^{T}$ is the control variable, $\mathbf{u}\left(k-\tau_{e}\right)=$ $\left[\begin{array}{llll}u_{1}\left(k-\tau_{1}\right) & u_{2}\left(k-\tau_{2}\right) & \cdots & u_{n}\left(k-\tau_{n}\right)\end{array}\right]^{T}$ is the control variable with lead times, $\mathbf{w}(k)$ is the customers' demands variable, $\mathbf{z}(k)$ is the total cost of the SC system, $\mathbf{A}_{i}$ is coefficients matrix of the inventory strategy implementation, $\mathbf{B}_{i}$ is coefficients matrix of the productivity and ordering placement, $\mathbf{B}_{i e}$ is coefficients matrix of the productivity and ordering placement during lead times, $\mathbf{B}_{w i}$ is coefficients matrix of customers' demands, $\mathbf{C}_{i}$ is coefficients matrix of the inventory cost, $\mathbf{D}_{i}$ is coefficients matrix of the manufacturing and ordering cost, and $\mathbf{D}_{i e}$ is coefficients matrix of the manufacturing and ordering cost with lead times.

This nonlinear SC system (2) is described with deviation values which are the differences between the actual values and the nominal values.

T-S fuzzy system is a powerful tool for processing nonlinear systems [23]. T-S fuzzy system consists of fuzzy rules that express local linear relationship between inputs and outputs of a system. Hence, based on T-S fuzzy system, we will establish a nonlinear SC fuzzy system.
For the nonlinear SC system (2), the $i$ th fuzzy control rule can be described as follows:

$$
R_{i} \text { : if } x_{1}(k) \text { is } M_{1}^{i} \text {, and, ..., and } x_{j}(k) \text { is } M_{j}^{i}, \ldots \text {, and } x_{n}(k)
$$
is $M_{n}^{i}$, then

$$
\begin{aligned}
\mathbf{x}(k+1)= & \mathbf{A}_{i} \mathbf{x}(k)+\mathbf{B}_{i} \mathbf{u}(k) \\
& +\sum_{e=1}^{n} \mathbf{B}_{i e} \mathbf{u}\left(k-\tau_{e}\right)+\mathbf{B}_{w i} \mathbf{w}(k), \\
\mathbf{z}(k)= & \mathbf{C}_{i} \mathbf{x}(k)+\mathbf{D}_{i} \mathbf{u}(k)+\sum_{e=1}^{n} \mathbf{D}_{i e} \mathbf{u}\left(k-\tau_{e}\right), \\
\mathbf{x}(k)= & \boldsymbol{\varphi}(k), \quad k=\{0,1, \ldots, N\},
\end{aligned}
$$

where $R_{i}(i=1,2, \ldots, r)$ is the $i$ th fuzzy rule, $r$ is the number of if-then rules, $M_{j}^{i}(j=1,2, \ldots, n)$ is the fuzzy set of the inventory level, and $\varphi(k)$ is the initial condition.

By singleton fuzzification, product inference, and centeraverage defuzzification, (3) can be inferred as

$$
\begin{aligned}
& \mathbf{x}(k+1)=\sum_{i=1}^{r} h_{i}(\mathbf{x}(k))\left[\mathbf{A}_{i} \mathbf{x}(k)+\mathbf{B}_{i} \mathbf{u}(k)\right. \\
& \left.+\sum_{e=1}^{n} \mathbf{B}_{i e} \mathbf{u}\left(k-\tau_{e}\right)+\mathbf{B}_{w i} \mathbf{w}(k)\right], \\
& \mathbf{z}(k)=\sum_{i=1}^{r} h_{i}(\mathbf{x}(k)) \\
& \cdot\left[\mathbf{C}_{i} \mathbf{x}(k)+\mathbf{D}_{i} \mathbf{u}(k)+\sum_{e=1}^{n} \mathbf{D}_{i e} \mathbf{u}\left(k-\tau_{e}\right)\right],
\end{aligned}
$$

where the membership function $h_{i}(\mathbf{x}(k))=\mu_{i}(\mathbf{x}(k)) /$ $\sum_{i=1}^{r} \mu_{i}(\mathbf{x}(k))$, in which $\mu_{i}(\mathbf{x}(k))=\prod_{j=1}^{n} M_{j}^{i}\left(x_{j}(k)\right) \cdot M_{j}^{i}\left(x_{j}(k)\right)$ represents the grade of membership of $x_{j}(k)$ in $M_{j}^{i}$. For all $k$, $h_{i}(\mathbf{x}(k)) \geq 0$ and $\sum_{i=1}^{r} h_{i}(\mathbf{x}(k))=1$. For simplicity, we omit $\mathbf{x}(k)$ in $h_{i}(\mathbf{x}(k))$.

2.2. Fuzzy Control Strategy. To restrain the impacts caused by lead times, switching actions among subsystems, and customers' stochastic demands on the nonlinear SC system, this paper will design a fuzzy robust $H_{\infty}$ control strategy. This strategy can incorporate the membership function of T$S$ fuzzy system into the robust $H_{\infty}$ control method to realize 
soft switching and make the system robustly asymptotically stable.

Based on the parallel distributed compensation scheme, the control law of the nonlinear SC system is formulated as follows.

Controller rule $\mathbf{K}_{i}$ is as follows: if $x_{1}(k)$ is $M_{1}^{i}$ and,..., and $x_{j}(k)$ is $M_{j}^{i}, \ldots$, and $x_{n}(k)$ is $M_{n}^{i}$, then

$$
\begin{gathered}
\mathbf{u}(k)=-\sum_{i=1}^{r} h_{i} \mathbf{K}_{i} \mathbf{x}(k), \\
\mathbf{u}\left(k-\tau_{e}\right)=-\sum_{i=1}^{r} h_{i} \mathbf{K}_{i e} \mathbf{x}\left(k-\tau_{e}\right),
\end{gathered}
$$

where $\mathbf{K}_{i}$ and $\mathbf{K}_{i e}$ denote the inventory feedback gains matrices of the $i$ th local model. $\mathbf{K}_{i}$ is the coefficients matrix of production plan and ordering delivery; $\mathbf{K}_{i e}$ is the coefficients matrix of production plan and ordering delivery during lead time.

Using the fuzzy controller (5), this paper intends to make the following system robustly asymptotically stable during lead times:

$$
\begin{gathered}
\mathbf{x}(k+1)=\sum_{i=1}^{r} \sum_{j=1}^{r} h_{i} h_{j}\left[\left(\mathbf{A}_{i}-\mathbf{B}_{i} \mathbf{K}_{j}\right) \mathbf{x}(k)\right. \\
\left.-\sum_{e=1}^{n} \mathbf{B}_{i e} \mathbf{K}_{j e} \mathbf{x}\left(k-\tau_{e}\right)+\mathbf{B}_{w i} \mathbf{w}(k)\right], \\
z(k)=\sum_{i=1}^{r} \sum_{j=1}^{r} h_{i} h_{j}\left[\left(\mathbf{C}_{i}-\mathbf{D}_{i} \mathbf{K}_{j}\right) \mathbf{x}(k)\right. \\
\left.-\sum_{e=1}^{n} \mathbf{D}_{i e} \mathbf{K}_{j e} \mathbf{x}\left(k-\tau_{e}\right)\right] .
\end{gathered}
$$

The inhibitory degree of this controller (5) can be described as the parameter $\gamma$; namely,

$$
\frac{\| \text { total } \operatorname{cost} \|_{2}}{\| \text { customers' demands } \|_{2}} \leq \gamma
$$

where $\|\cdot\|_{2}$ is $L_{2}$ norm [24]. The smaller the parameter $\gamma$ is, the better the performance of this SC fuzzy system (6) is.

2.3. Preliminaries. Before proceeding, we will introduce our theorem by recalling the following definitions, proposition, and lemmas.

Definition 2 (see [25]). For a given scalar $\gamma>0$ which denotes the disturbance attenuation level for a system, the nonlinear SC fuzzy system (6) is said to be robustly asymptotically stable with the $\gamma$ constraint under the $H_{\infty}$ norm if two conditions as below are satisfied.

(1) The nonlinear SC fuzzy system (6) is robustly asymptotically stable when $\mathbf{w}(k) \equiv 0$.

(2) Under zero-initial condition, the total cost $\mathbf{z}(k)$ of the nonlinear SC fuzzy system (6) satisfies $\|\mathbf{z}(k)\|_{2}^{2}<\gamma\|\mathbf{w}(k)\|_{2}^{2}$ for any nonzero $\mathbf{w}(k) \in \ell_{2}[0, \infty)$ and all admissible uncertainties.
Accordingly (5) is called a $\gamma$-suboptimal robust $H_{\infty}$ control law of the SC fuzzy system (6).

Definition 3 (see [26]). A cluster of fuzzy sets $\left\{F_{j}^{u}, u=\right.$ $\left.1,2, \ldots, q_{j}\right\}$ are said to be a standard fuzzy partition (SFP) in the universe $X$ if each $F_{j}^{u}$ is a normal fuzzy set and $F_{j}^{u}(u=$ $\left.1,2, \ldots, q_{j}\right)$ are full-overlapped in the universe $X . q_{j}$ is said to be the number of fuzzy partitions of the $j$ th input variable on $X$.

Definition 4 (see [26]). For a given fuzzy system, an overlapped-rules group with the largest amount of rules is said to be a maximal overlapped-rules group (MORG).

Proposition 5 (see [26]). If the input variables of a fuzzy system adopt SFPs, then all the rules in an overlapped-rules group must be included in a MORG.

Lemma 6 (see [27]). For any real matrices $\mathbf{X}_{i}, \mathbf{Y}_{i}$ for $1 \leq i \leq n$, and $\mathbf{S}>\mathbf{0}$ with appropriate dimensions, we have

$$
\begin{aligned}
2 \sum_{i=1}^{n} & \sum_{j=1}^{n} \sum_{k=1}^{n} \sum_{l=1}^{n} h_{i} h_{j} h_{k} h_{l} \mathbf{X}_{i j}^{T} \mathbf{S} \mathbf{Y}_{k l} \\
& \leq \sum_{i=1}^{n} \sum_{j=1}^{n} h_{i} h_{j}\left(\mathbf{X}_{i j}^{T} \mathbf{S} \mathbf{X}_{i j}+\mathbf{Y}_{i j}^{T} \mathbf{S} \mathbf{Y}_{i j}\right),
\end{aligned}
$$

where $h_{i}(1 \leq i \leq n)$ are defined as $h_{i}(M(k)) \geq 0$, $\sum_{i=1}^{n} h_{i}(M(k))=1$.

Lemma 7. For any real matrices $\mathbf{X}_{i j}(1 \leq i, j \leq n)$, and $\mathbf{S}>\mathbf{0}$ with appropriate dimensions, the following inequality holds:

$$
\sum_{i=1}^{n} \sum_{j=1}^{n} \sum_{k=1}^{n} \sum_{l=1}^{n} h_{i} h_{j} h_{k} h_{l} \mathbf{X}_{i j}^{T} \mathbf{S} \mathbf{X}_{k l} \leq \sum_{i=1}^{n} \sum_{j=1}^{n} h_{i} h_{j} \mathbf{X}_{i j}^{T} \mathbf{S} \mathbf{X}_{i j} .
$$

Proof. For Lemma 6, let $\mathbf{X}=\mathbf{Y}$; then we have

$$
\begin{aligned}
2 \sum_{i=1}^{n} & \sum_{j=1}^{n} \sum_{k=1}^{n} \sum_{l=1}^{n} h_{i} h_{j} h_{k} h_{l} \mathbf{X}_{i j}^{T} \mathbf{S} \mathbf{X}_{k l} \\
& \leq \sum_{i=1}^{n} \sum_{j=1}^{n} h_{i} h_{j}\left(\mathbf{X}_{i j}^{T} \mathbf{S} \mathbf{X}_{i j}+\mathbf{X}_{i j}^{T} \mathbf{S} \mathbf{X}_{i j}\right) \\
& =2 \sum_{i=1}^{n} \sum_{j=1}^{n} h_{i} h_{j} \mathbf{X}_{i j}^{T} \mathbf{S} \mathbf{X}_{i j} .
\end{aligned}
$$

Therefore, $\quad \sum_{i=1}^{n} \sum_{j=1}^{n} \sum_{k=1}^{n} \sum_{l=1}^{n} h_{i} h_{j} h_{k} h_{l} \mathbf{X}_{i j}^{T} \mathbf{S} \mathbf{X}_{k l} \leq$ $\sum_{i=1}^{n} \sum_{j=1}^{n} h_{i} h_{j} \mathbf{X}_{i j}^{T} \mathbf{S} \mathbf{X}_{i j}$ can be obtained.

\section{Fuzzy Robust $H_{\infty}$ Control of Nonlinear SC}

A fuzzy robust $H_{\infty}$ output-feedback controller for the T-S fuzzy system with uncertainties was recently introduced by [28]. It also came to use in [29] to restraint of the bullwhip effect for uncertain closed-loop SC system.

In this section, we also apply this idea of the fuzzy controller for the nonlinear SC fuzzy system (6) with lead times. 
Theorem 8. For a given scalar $\gamma>0$, if there exist local common positive definite matrices $\mathbf{P}_{c}$ and $\mathbf{Q}_{e c}$ in $\mathbf{G}_{c}$ such that the following linear matrix inequalities (LMIs) (11) and (12) are satisfied, then the supply chain fuzzy system (6) with lead times and SFP inputs is robustly asymptotically stable and the $H_{\infty}$ norm is less than a given bound $\gamma$ :

$$
\begin{aligned}
& {\left[\begin{array}{ccc}
-\overline{\overline{\mathbf{P}}} & * & * \\
\overline{\mathbf{M}}_{i i} & -\mathbf{P}_{c}^{-1} & * \\
\overline{\mathbf{N}}_{i i} & \mathbf{0} & -\mathbf{I}
\end{array}\right]<\mathbf{0}, \quad i \in I_{c},} \\
& {\left[\begin{array}{ccc}
-4 \overline{\overline{\mathbf{P}}} & * & * \\
2 \overline{\overline{\mathbf{M}}}_{i j} & -\mathbf{P}_{c}^{-1} & * \\
2 \overline{\overline{\mathbf{N}}}_{i j} & \mathbf{0} & -\mathbf{I}
\end{array}\right]<\mathbf{0}, \quad i<j, i, j \in I_{c},}
\end{aligned}
$$

where $\overline{\overline{\mathbf{P}}}=\left[\begin{array}{cccc}\mathbf{P}_{c}-\sum_{e=1}^{n} \mathbf{Q}_{e c} & * & * \\ \mathbf{0} & \widehat{\mathbf{Q}} & * \\ \mathbf{0} & \mathbf{0} & \gamma^{2} \mathbf{I}\end{array}\right], \widehat{\mathbf{Q}}=\operatorname{diag}\left\{\begin{array}{lllll}\mathbf{Q}_{1 c} & \cdots & \mathbf{Q}_{e c} & \cdots & \mathbf{Q}_{n c}\end{array}\right\}$, $\overline{\mathbf{M}}_{i j}=\left[\begin{array}{lllllll}\mathbf{M}_{i j} & -\mathbf{B}_{i 1} \mathbf{K}_{j 1 c} & \cdots & -\mathbf{B}_{i e} \mathbf{K}_{j e c} & \cdots & -\mathbf{B}_{i n} \mathbf{K}_{j n c} & \mathbf{B}_{w i}\end{array}\right]$, $\mathbf{M}_{i j} \quad=\quad \mathbf{A}_{i}-\mathbf{B}_{i} \mathbf{K}_{j c}, \quad \overline{\mathbf{N}}_{i j}=$

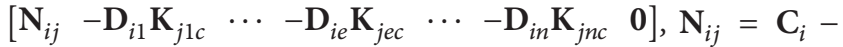
$\mathbf{D}_{i} \mathbf{K}_{j c}, \overline{\overline{\mathbf{M}}}_{i j}=\left(\overline{\mathbf{M}}_{i j}+\overline{\mathbf{M}}_{j i}\right) / 2, \overline{\overline{\mathbf{N}}}_{i j}=\left(\overline{\mathbf{N}}_{i j}+\overline{\mathbf{N}}_{j i}\right) / 2, \mathbf{0}$ denotes the zero matrix, I denotes the identity matrix, $I_{c}$ is a set of the rule numbers included in $\mathbf{G}_{c}, \mathbf{G}_{c}$ denotes the cth $M O R G$, $c=1,2, \ldots, \prod_{j=1}^{n}\left(m_{j}-1\right)$, and $m_{j}$ is the number of the fuzzy partitions of the $j$ th input variable.

Proof. Consider two scenarios: first, if state input variables $\mathbf{x}(k)$ and $\mathbf{x}(k+1)$ are in the same overlapped-rules group, the fuzzy system (6) will be proved to be robustly asymptotically stable. Then if state input variables $\mathbf{x}(k)$ and $\mathbf{x}(k+1)$ are in the different overlapped-rules groups, the same result will be obtained.

Assume that the fuzzy system (6) contains $f$ overlappedrules groups; $v_{d}(d=1,2, \ldots, f)$ is the operating region of the $d$ th overlapped-rules group and $L_{d}=$ the rule numbers included in the $d$ th overlapped-rules group\}.

In the first scenario, the local model of the $d$ th overlapped-rules group can be described as

$$
\begin{gathered}
\mathbf{x}(k+1)=\sum_{i \in L_{d}} \sum_{j \in L_{d}} h_{i} h_{j} \\
\cdot\left[\mathbf{M}_{i j} \mathbf{x}(k)-\sum_{e=1}^{n} \mathbf{B}_{i e} \mathbf{K}_{j e c} \mathbf{x}\left(k-\tau_{e}\right)+\mathbf{B}_{w i} \mathbf{w}(k)\right], \\
\mathbf{z}(k)=\sum_{i \in L_{d}} \sum_{j \in L_{d}} h_{i} h_{j}\left[\mathbf{N}_{i j} \mathbf{x}(k)-\sum_{e=1}^{n} \mathbf{D}_{i e} \mathbf{K}_{j e c} \mathbf{x}\left(k-\tau_{e}\right)\right],
\end{gathered}
$$

where $\mathbf{K}_{j e c}$ is the state feedback control gain of production lead time and ordering lead time in the $c$ th MORG.
Equation (13) can be represented as follows:

$$
\begin{aligned}
\mathbf{x}(k+1) & =\sum_{i \in L_{d}} \sum_{j \in L_{d}} h_{i} h_{j} \overline{\mathbf{M}}_{i j} \overline{\mathbf{x}}(k), \\
z(k) & =\sum_{i \in L_{d}} \sum_{j \in L_{d}} h_{i} h_{j} \overline{\mathbf{N}}_{i j} \overline{\mathbf{x}}(k),
\end{aligned}
$$

where

$\overline{\mathbf{x}}(k)$

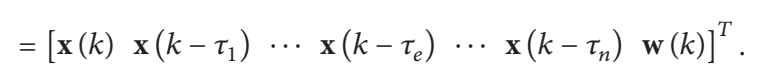

Consider the discrete Lyapunov function:

$$
V_{d}(\mathbf{x}(k))=\mathbf{x}^{T}(k) \mathbf{P}_{c} \mathbf{x}(k)+\sum_{e=1}^{n} \sum_{\xi=k-\tau_{e}}^{k-1} \mathbf{x}^{T}(\xi) \mathbf{Q}_{e c} \mathbf{x}(\xi) .
$$

And using Lemma 7 will supply

$$
\begin{aligned}
& \Delta V_{d}(\mathbf{x}(k))=V_{d}(\mathbf{x}(k+1))-V_{d}(\mathbf{x}(k))=\mathbf{x}^{T}(k+1) \\
& \cdot \mathbf{P}_{c} \mathbf{x}(k+1)-\mathbf{x}^{T}(k) \mathbf{P}_{c} \mathbf{x}(k) \\
& +\sum_{e=1}^{n}\left[\mathbf{x}^{T}(k) \mathbf{Q}_{e c} \mathbf{x}(k)-\mathbf{x}^{T}\left(k-\tau_{e}\right) \mathbf{Q}_{e c} \mathbf{x}\left(k-\tau_{e}\right)\right] \\
& =\sum_{i \in L_{d}} \sum_{j \in L_{d}} h_{i} h_{j} \sum_{p \in L_{d}} \sum_{q \in L_{d}} h_{p} h_{q} \\
& \cdot\left[\overline{\mathbf{x}}^{T}(k) \overline{\mathbf{M}}_{i j}^{T} \mathbf{P}_{c} \overline{\mathbf{M}}_{p q} \overline{\mathbf{x}}(k)-\mathbf{x}^{T}(k) \mathbf{P}_{c} \mathbf{x}(k)\right] \\
& +\sum_{e=1}^{n}\left[\mathbf{x}^{T}(k) \mathbf{Q}_{e c} \mathbf{x}(k)-\mathbf{x}^{T}\left(k-\tau_{e}\right) \mathbf{Q}_{e c} \mathbf{x}\left(k-\tau_{e}\right)\right] \\
& =\sum_{i \in L_{d}} \sum_{j \in L_{d}} h_{i} h_{j} \sum_{p \in L_{d}} \sum_{q \in L_{d}} h_{p} h_{q} \overline{\mathbf{x}}^{T}(k)\left(\overline{\mathbf{M}}_{i j}^{T} \mathbf{P}_{c} \overline{\mathbf{M}}_{p q}-\overline{\mathbf{P}}\right) \\
& \cdot \overline{\mathbf{x}}(k)=\sum_{i \in L_{d}} \sum_{j \in L_{d}} h_{i} h_{j} \sum_{p \in L_{d}} \sum_{q \in L_{d}} h_{p} h_{q} \overline{\mathbf{x}}^{T}(k) \\
& \cdot\left[\left(\frac{\overline{\mathbf{M}}_{i j}+\overline{\mathbf{M}}_{j i}}{2}\right)^{T} \mathbf{P}_{c}\left(\frac{\overline{\mathbf{M}}_{p q}+\overline{\mathbf{M}}_{q p}}{2}\right)-\overline{\mathbf{P}}\right] \overline{\mathbf{x}}(k) \\
& =\sum_{i \in L_{d}} \sum_{j \in L_{d}} h_{i} h_{j} \sum_{p \in L_{d}} \sum_{q \in L_{d}} h_{p} h_{q} \overline{\mathbf{x}}^{T}(k) \\
& \cdot\left(\overline{\overline{\mathbf{M}}}_{i j}^{T} \mathbf{P}_{c} \overline{\overline{\mathbf{M}}}_{p q}-\overline{\mathbf{P}}\right) \overline{\mathbf{x}}(k) \leq \sum_{i \in L_{d}} \sum_{j \in L_{d}} h_{i} h_{j} \overline{\mathbf{x}}^{T}(k) \\
& \cdot\left(\overline{\overline{\mathbf{M}}}_{i j}^{T} \mathbf{P}_{c} \overline{\overline{\mathbf{M}}}_{i j}-\overline{\mathbf{P}}\right) \overline{\mathbf{x}}(k),
\end{aligned}
$$

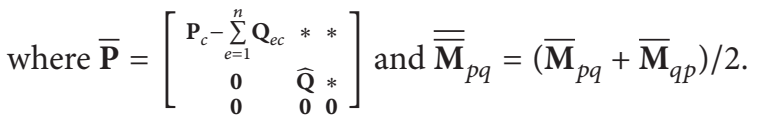


Thus $\Delta V_{d}(\mathbf{x}(k))$ satisfies the relation

$$
\begin{aligned}
& \Delta V_{d}(\mathbf{x}(k)) \\
& \leq \sum_{i=j, i \in L_{d}} h_{i}^{2} \overline{\mathbf{x}}^{T}(k)\left[\overline{\mathbf{M}}_{i i}^{T} \mathbf{P}_{c} \overline{\mathbf{M}}_{i i}-\overline{\mathbf{P}}\right] \overline{\mathbf{x}}(k) \\
& \quad+2 \sum_{\substack{i<j \\
i \in L_{d}, j \in L_{d}}} h_{i} h_{j} \overline{\mathbf{x}}^{T}(k)\left[\overline{\mathbf{M}}_{i j}^{T} \mathbf{P}_{c} \overline{\overline{\mathbf{M}}}_{i j}-\overline{\mathbf{P}}\right] \overline{\mathbf{x}}(k) .
\end{aligned}
$$

Assume the customers' demands $\mathbf{w}(k) \neq \mathbf{0} ; H_{\infty}$ performance index function $J_{1}$ can be presented by

$$
J_{1}=\sum_{k=0}^{N-1}\left[z^{T}(k) z(k)-\gamma^{2} \mathbf{w}^{T}(k) \mathbf{w}(k)\right] .
$$

The above equation can be rewritten as

$J_{1}$

$$
\begin{aligned}
= & \sum_{k=0}^{N-1}\left[z^{T}(k) z(k)-\gamma^{2} \mathbf{w}^{T}(k) \mathbf{w}(k)+\Delta V_{d}(\mathbf{x}(k))\right] \\
& -V_{d}(\mathbf{x}(N)) \\
\leq & \sum_{k=0}^{N-1}\left[z^{T}(k) z(k)-\gamma^{2} \mathbf{w}^{T}(k) \mathbf{w}(k)+\Delta V_{d}(\mathbf{x}(k))\right] .
\end{aligned}
$$

Substituting (18) into (20), we have

$$
\begin{aligned}
J_{1} & \leq \sum_{k=0}^{N-1}\left\{\sum_{i=j, i \in L_{d}} h_{i}^{2} \overline{\mathbf{x}}^{T}(k)\left[\overline{\mathbf{M}}_{i i}^{T} \mathbf{P}_{c} \overline{\mathbf{M}}_{i i}-\overline{\overline{\mathbf{P}}}+\overline{\mathbf{N}}_{i i}^{T} \overline{\mathbf{N}}_{i i}\right]\right. \\
& \cdot \overline{\mathbf{x}}(k)\}+2 \sum_{k=0}^{N-1}\left\{\sum_{\substack{i<j, i \in L_{d} \\
j \in L_{d}}} h_{i} h_{j} \overline{\mathbf{x}}^{T}(k)\right. \\
& \left.\cdot\left[\overline{\mathbf{M}}_{i j}^{T} \mathbf{P}_{c} \overline{\overline{\mathbf{M}}}_{i j}-\overline{\overline{\mathbf{P}}}+\overline{\overline{\mathbf{N}}}_{i j}^{T} \overline{\overline{\mathbf{N}}}_{i j}\right] \overline{\mathbf{x}}(k)\right\} .
\end{aligned}
$$

Applying Schur complement to (11) and (12) results in $\overline{\mathbf{M}}_{i i}^{T} \mathbf{P}_{c} \overline{\mathbf{M}}_{i i}-\overline{\overline{\mathbf{P}}}+\overline{\mathbf{N}}_{i i}^{T} \overline{\mathbf{N}}_{i i}<\mathbf{0}$ and $\overline{\overline{\mathbf{M}}}_{i j}^{T} \mathbf{P}_{c} \overline{\overline{\mathbf{M}}}_{i j}-\overline{\overline{\mathbf{P}}}+\overline{\overline{\mathbf{N}}}_{i j}^{T} \overline{\overline{\mathbf{N}}}_{i j}<\mathbf{0}$. Then, $J_{1}<0$ can be obtained; that is, $z^{T}(k) z(k)<\gamma^{2} \mathbf{w}^{T}(k) \mathbf{w}(k)$; moreover, let $N \rightarrow+\infty$; then we have $\|z(k)\|_{2}^{2}<\gamma^{2}\|\mathbf{w}(k)\|_{2}^{2}$. As a result, the SC system (14) is proved to be asymptotically stable in the case of $\mathbf{w}(k) \neq \mathbf{0}$.

On the other hand, if $\mathbf{w}(k) \equiv \mathbf{0}$, it is obvious that (18) is equivalent to the following inequality:

$$
\begin{aligned}
& \Delta V_{d}(\mathbf{x}(k)) \\
& \leq \sum_{i=j, i \in L_{d}} h_{i}^{2} \overline{\mathbf{x}}^{T}(k)\left[\overline{\mathbf{M}}_{i i}^{T} \mathbf{P}_{c} \overline{\mathbf{M}}_{i i}-\overline{\overline{\mathbf{P}}}\right] \overline{\mathbf{x}}(k) \\
& \quad+2 \sum_{\substack{i<j \\
i \in L_{d}, j \in L_{d}}} h_{i} h_{j} \overline{\mathbf{x}}^{T}(k)\left[\overline{\overline{\mathbf{M}}}_{i j}^{T} \mathbf{P}_{c} \overline{\overline{\mathbf{M}}}_{i j}-\overline{\overline{\mathbf{P}}}\right] \overline{\mathbf{x}}(k) .
\end{aligned}
$$

According to (11) and (12) we can obtain $\overline{\mathbf{M}}_{i i}^{T} \mathbf{P}_{c} \overline{\mathbf{M}}_{i i}-$ $\overline{\overline{\mathbf{P}}}<\mathbf{0}$ and $\overline{\overline{\mathbf{M}}}_{i j}^{T} \mathbf{P}_{c} \overline{\overline{\mathbf{M}}}_{i j}-\overline{\overline{\mathbf{P}}}<\mathbf{0}$, respectively. Accordingly, we can conclude that $\Delta V_{d}(\mathbf{x}(k))<0$. As a result, the state feedback controller can ensure the local system (6) robustly asymptotically stable in the $d$ th overlapped-rules group.

In the second scenario: a characteristic function in any overlapped-rules group is constructed as follows:

$$
\lambda_{d}= \begin{cases}1, & \mathbf{x}(k) \in v_{d} \\ 0, & \mathbf{x}(k) \notin v_{d},\end{cases}
$$

where $\sum_{d=1}^{f} \lambda_{d}=1$; then the global model of the discrete fuzzy system in the input universe of the discourse can be described as follows:

$$
\begin{array}{r}
\mathbf{x}(k+1)=\sum_{d=1}^{f} \lambda_{d}\left[\sum_{i \in L_{d}} \sum_{j \in L_{d}} h_{i} h_{j} \overline{\mathbf{M}}_{i j} \overline{\mathbf{x}}(k)\right], \\
z(k)=\sum_{d=1}^{f} \lambda_{d}\left[\sum_{i \in L_{d}} \sum_{j \in L_{d}} h_{i} h_{j} \overline{\mathbf{N}}_{i j} \overline{\mathbf{x}}(k)\right] .
\end{array}
$$

In the following, based on the definitions of $\mathbf{P}_{m}=$ $\sum_{d=1}^{f} \lambda_{d} \mathbf{P}_{c}$ and $\mathbf{Q}_{e m}=\sum_{d=1}^{f} \lambda_{d} \mathbf{Q}_{e c}$, a piecewise Lyapunov function can be introduced in the input universe of the discourse as follows:

$$
\begin{aligned}
V(\mathbf{x}(k))=\mathbf{x}^{T}(k) \mathbf{P}_{m} \mathbf{x}(k)+\sum_{e=1}^{n} \sum_{\xi=k-\tau_{e}}^{k-1} \mathbf{x}^{T}(\xi) \mathbf{Q}_{e m} \mathbf{x}(\xi) \\
=\mathbf{x}^{T}(k)\left(\sum_{d=1}^{f} \lambda_{d} \mathbf{P}_{c}\right) \mathbf{x}(k) \\
\quad+\sum_{e=1}^{n} \sum_{\xi=k-\tau_{e}}^{k-1} \mathbf{x}^{T}(\xi)\left(\sum_{d=1}^{f} \lambda_{d} \mathbf{Q}_{e c}\right) \mathbf{x}(\xi) \\
=\sum_{d=1}^{f} \lambda_{d}\left[\mathbf{x}^{T}(k) \mathbf{P}_{c} \mathbf{x}(k)+\sum_{e=1}^{n} \sum_{\xi=k-\tau_{e}}^{k-1} \mathbf{x}^{T}(\xi) \mathbf{Q}_{e c} \mathbf{x}(\xi)\right] \\
=\sum_{d=1}^{f} \lambda_{d} V_{d}(\mathbf{x}(k)) .
\end{aligned}
$$

We first assume that the customers' demands $\mathbf{w}(k) \neq$ 0. For (24) we can obtain $J_{2}=\sum_{k=0}^{N-1} \sum_{d=1}^{f} \lambda_{d}\left[z^{T}(k) z(k)-\right.$ $\left.\gamma^{2} \mathbf{w}^{T}(k) \mathbf{w}(k)\right]$ after the $H_{\infty}$ performance index function $J_{1}=\sum_{k=0}^{N-1}\left[z^{T}(k) z(k)-\gamma^{2} \mathbf{w}^{T}(k) \mathbf{w}(k)\right]$ is considered. $J_{2}<$ 0 can be obtained through a similar procedure; that is, $z^{T}(k) z(k)<\gamma^{2} \mathbf{w}^{T}(k) \mathbf{w}(k)$; moreover, let $N \rightarrow+\infty$; then we have $\|z(k)\|_{2}^{2}<\gamma^{2}\|\mathbf{w}(k)\|_{2}^{2}$. As a result, (24) is proved to be asymptotically stable in the case of $\mathbf{w}(k) \neq \mathbf{0}$.

On the other hand, if $\mathbf{w}(k) \equiv \mathbf{0}$, we have

$$
\begin{aligned}
\Delta V(\mathbf{x}(k)) & =V(\mathbf{x}(k+1))-V(\mathbf{x}(k)) \\
& =\sum_{d=1}^{f} \lambda_{d} V_{d}(\mathbf{x}(k+1))-\sum_{d=1}^{f} \lambda_{d} V_{d}(\mathbf{x}(k))
\end{aligned}
$$




$$
\begin{aligned}
& =\sum_{d=1}^{f} \lambda_{d}\left[V_{d}(\mathbf{x}(k+1))-V_{d}(\mathbf{x}(k))\right] \\
& =\sum_{d=1}^{f} \lambda_{d} \Delta V_{d}(\mathbf{x}(k))<0 .
\end{aligned}
$$

Hence, in any overlapped-rules group, (24) with $\mathbf{w}(k) \equiv \mathbf{0}$ is asymptotically stable by the fuzzy controller (5).

Therefore, according to Proposition 5, we can conclude that the fuzzy system (6) is robustly asymptotically stable with Condition (11) and Condition (12) by resorting to find local common positive definite matrices $\mathbf{P}_{c}$ and $\mathbf{Q}_{e c}$ in $\mathbf{G}_{c}$. This completes the proof of the theorem.

In Theorem 8, for a given $H_{\infty}$ performance index $\gamma$, we can obtain the robust stabilization conditions of (6), which are represented by a set of matrix inequalities in (11) and (12). Subsequently we will show that such inequalities can be transformed into LMIs when designing the actual $H_{\infty}$ controllers. Note that the feasibility of LMIs can be easily achieved by using the LMI toolbox in MATLAB.

Theorem 8 can be recast as the LMI problem by the following Theorem 9.
Theorem 9. For the supply chain fuzzy system (6) with lead times and SFP inputs, if there exist a given scalar $\gamma>0$, local common positive definite matrices $\mathbf{P}_{c}$ and $\mathbf{Q}_{e c}$, and matrices $\mathbf{K}_{i c}, \mathbf{K}_{j c}, \mathbf{K}_{i e c}, \mathbf{K}_{j e c}$ in $\mathbf{G}_{c}$, such that the following LMIs are satisfied, then the supply chain fuzzy system (6) is robustly asymptotically stable under the performance $\gamma$ :

$$
\begin{aligned}
& {\left[\begin{array}{ccccc}
-\mathbf{P}_{c}+\sum_{e=1}^{n} \mathbf{Q}_{e c} & * & * & * & * \\
\mathbf{0} & -\widehat{\mathbf{Q}} & * & * & * \\
\mathbf{0} & \mathbf{0} & -\gamma^{2} \mathbf{I} & * & * \\
\mathbf{A}_{i}-\mathbf{B}_{i} \mathbf{K}_{i c} & -\boldsymbol{\Pi}_{1} & \mathbf{B}_{w i} & -\mathbf{P}_{c} & * \\
\mathbf{C}_{i}-\mathbf{D}_{i} \mathbf{K}_{i c} & -\Pi_{2} & \mathbf{0} & \mathbf{0} & -\mathbf{I}
\end{array}\right]<\mathbf{0}, i \in I_{c},} \\
& {\left[\begin{array}{cccccc}
-4 \mathbf{P}_{c}+4 \sum_{e=1}^{n} \mathbf{Q}_{e c} & * & * & * & * \\
\mathbf{0} & -4 \widehat{\mathbf{Q}} & * & * & * \\
\mathbf{0} & \mathbf{0} & -4 \gamma^{2} \mathbf{I} & * & * \\
\mathbf{A}_{i}-\mathbf{B}_{i} \mathbf{K}_{j c}+\mathbf{A}_{j}-\mathbf{B}_{j} \mathbf{K}_{i c} & -\boldsymbol{\Phi}_{1} & \mathbf{B}_{w i}+\mathbf{B}_{w j} & -\mathbf{P}_{c} & * \\
\mathbf{C}_{i}-\mathbf{D}_{i} \mathbf{K}_{j c}+\mathbf{C}_{j}-\mathbf{D}_{j} \mathbf{K}_{i c} & -\boldsymbol{\Phi}_{2} & \mathbf{0} & \mathbf{0} & -\mathbf{I}
\end{array}\right]} \\
& <\mathbf{0}, \quad i<j, i, j \in I_{c}, \\
& <
\end{aligned}
$$

where

$$
\begin{aligned}
\widehat{\mathbf{Q}} & =\operatorname{diag}\left\{\begin{array}{lllll}
\mathbf{Q}_{1 c} & \cdots & \mathbf{Q}_{e c} & \cdots & \mathbf{Q}_{n c}
\end{array}\right\}, \\
\boldsymbol{\Pi}_{1} & =\left[\begin{array}{lllll}
\mathbf{B}_{i 1} \mathbf{K}_{i 1 c} & \cdots & \mathbf{B}_{i e} \mathbf{K}_{i e c} & \cdots & \mathbf{B}_{i n} \mathbf{K}_{i n c}
\end{array}\right], \\
\boldsymbol{\Pi}_{2} & =\left[\begin{array}{lllll}
\mathbf{D}_{i 1} \mathbf{K}_{i 1 c} & \cdots & \mathbf{D}_{i e} \mathbf{K}_{i e c} & \cdots & \mathbf{D}_{i n} \mathbf{K}_{i n c}
\end{array}\right], \\
\boldsymbol{\Phi}_{1} & =\left[\begin{array}{lllll}
\mathbf{B}_{i 1} \mathbf{K}_{j 1 c}+\mathbf{B}_{j 1} \mathbf{K}_{i 1 c} & \cdots & \mathbf{B}_{i e} \mathbf{K}_{j e c}+\mathbf{B}_{j e} \mathbf{K}_{i e c} & \cdots & \mathbf{B}_{i n} \mathbf{K}_{j n c}+\mathbf{B}_{j n} \mathbf{K}_{i n c}
\end{array}\right], \\
\boldsymbol{\Phi}_{2} & =\left[\begin{array}{lllll}
\mathbf{D}_{i 1} \mathbf{K}_{j 1 c}+\mathbf{D}_{j 1} \mathbf{K}_{i 1 c} & \cdots & \mathbf{D}_{i e} \mathbf{K}_{j e c}+\mathbf{D}_{j e} \mathbf{K}_{i e c} & \cdots & \mathbf{D}_{i n} \mathbf{K}_{j n c}+\mathbf{D}_{j n} \mathbf{K}_{i n c}
\end{array}\right],
\end{aligned}
$$

$I_{c}$ is a set of the rule numbers included in $\mathbf{G}_{c}, \mathbf{G}_{c}$ denotes the cth MORG, $c=1,2, \ldots, \prod_{j=1}^{n}\left(m_{j}-1\right)$, and $m_{j}$ is the number of the fuzzy partitions of the jth input variable.

Proof. The proof is analogous to that of Theorem 8. For simplicity, the similar sections are truncated. Theorem 9 can be easily demonstrated by using matrix transformations and the Schur complement. The main procedure is as follows.

In order to solve the LMIs, (11) can be expressed further as follows:

$$
\left[\begin{array}{ccccc}
-\mathbf{P}_{c}+\sum_{e=1}^{n} \mathbf{Q}_{e c} & * & * & * & * \\
\mathbf{0} & -\widehat{\mathbf{Q}} & * & * & * \\
\mathbf{0} & \mathbf{0} & -\gamma^{2} \mathbf{I} & * & * \\
\mathbf{A}_{i}-\mathbf{B}_{i} \mathbf{K}_{i c} & -\Pi_{1} & \mathbf{B}_{w i} & -\mathbf{P}_{c}^{-1} & * \\
\mathbf{C}_{i}-\mathbf{D}_{i} \mathbf{K}_{i c} & -\boldsymbol{\Pi}_{2} & \mathbf{0} & \mathbf{0} & -\mathbf{I}
\end{array}\right]<\mathbf{0} .
$$

Taking the congruence transformation with $\operatorname{diag}\{\mathbf{I}, \mathbf{I}, \mathbf{I}$, $\left.\mathbf{P}_{c}, \mathbf{I}\right\}$ easily verifies

$$
\left[\begin{array}{ccccc}
-\mathbf{P}_{c}+\sum_{e=1}^{n} \mathbf{Q}_{e c} & * & * & * & * \\
\mathbf{0} & -\widehat{\mathbf{Q}} & * & * & * \\
\mathbf{0} & \mathbf{0} & -\gamma^{2} \mathbf{I} & * & * \\
\mathbf{A}_{i}-\mathbf{B}_{i} \mathbf{K}_{i c} & -\boldsymbol{\Pi}_{1} & \mathbf{B}_{w i} & -\mathbf{P}_{c} & * \\
\mathbf{C}_{i}-\mathbf{D}_{i} \mathbf{K}_{i c} & -\boldsymbol{\Pi}_{2} & \mathbf{0} & \mathbf{0} & -\mathbf{I}
\end{array}\right]<\mathbf{0} .
$$

Clearly, (12) is equivalent to

$$
\left[\begin{array}{ccccc}
4\left(-\mathbf{P}_{c}+\sum_{e=1}^{n} \mathbf{Q}_{e c}\right) & * & * & * & * \\
\mathbf{0} & -4 \widehat{\mathbf{Q}} & * & * & * \\
\mathbf{0} & \mathbf{0} & -4 \gamma^{2} \mathbf{I} & * & * \\
\mathbf{A}_{i}-\mathbf{B}_{i} \mathbf{K}_{j c}+\mathbf{A}_{j}-\mathbf{B}_{j} \mathbf{K}_{i c} & -\boldsymbol{\Phi}_{1} & \mathbf{B}_{w i}+\mathbf{B}_{w j} & -\mathbf{P}_{c}^{-1} & * \\
\mathbf{C}_{i}-\mathbf{D}_{i} \mathbf{K}_{j c}+\mathbf{C}_{j}-\mathbf{D}_{j} \mathbf{K}_{i c} & -\boldsymbol{\Phi}_{2} & \mathbf{0} & \mathbf{0} & -\mathbf{I}
\end{array}\right]
$$


TABLE 1: The comparison results of the numbers of LMIs satisfied between Theorem 3 in [30] and Theorem 9 in this paper.

\begin{tabular}{ccccc}
\hline$n$ & $m$ & $r$ & $v$ & 9 \\
\hline 1 & 3 & 3 & 29 & 18 \\
1 & 4 & 4 & 53 & 27 \\
2 & 3 & 9 & 428 & 64 \\
2 & 4 & 16 & 2213 & 144 \\
\hline
\end{tabular}

Taking the congruence transformation with $\operatorname{diag}\{\mathbf{I}, \mathbf{I}, \mathbf{I}$, $\left.\mathbf{P}_{c}, \mathbf{I}\right\}$ results in

$$
\begin{aligned}
& {\left[\begin{array}{ccccc}
-4 \mathbf{P}_{c}+4 \sum_{e=1}^{n} \mathbf{Q}_{e c} & * & * & * & * \\
\mathbf{0} & -4 \widehat{\mathbf{Q}} & * & * & * \\
\mathbf{0} & \mathbf{0} & -4 \gamma^{2} \mathbf{I} & * & * \\
\mathbf{A}_{i}-\mathbf{B}_{i} \mathbf{K}_{j c}+\mathbf{A}_{j}-\mathbf{B}_{j} \mathbf{K}_{i c} & -\boldsymbol{\Phi}_{1} & \mathbf{B}_{w i}+\mathbf{B}_{w j} & -\mathbf{P}_{c} & * \\
\mathbf{C}_{i}-\mathbf{D}_{i} \mathbf{K}_{j c}+\mathbf{C}_{j}-\mathbf{D}_{j} \mathbf{K}_{i c} & -\boldsymbol{\Phi}_{2} & \mathbf{0} & \mathbf{0} & -\mathbf{I}
\end{array}\right]} \\
& <\mathbf{0} .
\end{aligned}
$$

Remark 10. (1) Compared with the common robust control approach, the proposed fuzzy robust control strategy is less sensitive to the variations of system parameters and can realize soft switching and make the system robustly asymptotically stable. (2) The proposed fuzzy robust control strategy requires only finding local common positive definite matrices in each MORG to check the robust stability of T-S fuzzy system. Therefore, the new control strategy can reduce the conservation and difficulty of the common Lyapunov function approach. (3) The performance and validity of the controller (5) depend on the parameter $\gamma$, which will play an important role in the existence of some matrices satisfying a series of LMIs. The smaller the parameter $\gamma$ is, the better the performance of the system will be. Therefore, when $\gamma$ is set too small in order to obtain the better performance of the system, some matrices satisfying a series of LMIs may not exist. For a smaller $\gamma$, if some matrices satisfying a series of LMIs do not exist, $\gamma$ will be constantly set to a bigger value little by little until some matrices satisfying a series of LMIs exist.

Remark 11. The numbers of LMIs satisfied to check the stability of a system for Theorem 9 in this paper and for Theorem 3 in [30] are $\prod_{j=1}^{n}\left(m_{j}-1\right) 2^{(n-1)}\left(2^{n}+1\right)+6 \prod_{j=1}^{n}\left(m_{j}-\right.$ $1)$ and $(1 / 2)\left(\prod_{j=1}^{n}\left(m_{j}\right)\right)^{3}+(1 / 2)\left(\prod_{j=1}^{n}\left(m_{j}\right)\right)^{2}+2 \prod_{j=1}^{n}\left(m_{j}\right)+5$, respectively.

Table 1 shows the comparison results of the numbers of LMIs satisfied between Theorem 3 in [30] and Theorem 9 in this paper, where $n$ denotes the number of state variables and $v$ and $\vartheta$ denote the numbers of LMIs satisfied for Theorem 3 in [30] and Theorem 9 in this paper, respectively.

From Table 1, we know that the difference of $v$ and $\vartheta$ becomes more and more large along with the increase of $r$.

\section{Simulation Research}

4.1. Modeling of Two-Stage Chain-Type SC Fuzzy System. We illustrate the effectiveness of the fuzzy robust $H_{\infty}$ control strategy described in Section 3, which is based on Chinese compressors manufacturing industry. This industry can be considered as a two-stage chain-type nonlinear SC system with production lead time and ordering lead time.

The manufacturer's production strategies are set as follows. (1) When the manufacturer's inventory level $x_{1}(k)<$ 0 , the manufacturer manufactures the products according to the JIT (Just In Time) mode in order to meet the retailer's order needs. (2) When $x_{1}(k) \in\left[0, I_{0 m}\right]\left(I_{0 m}\right.$ is the manufacturer's expected inventory), the manufacturer normally manufactures the products to satisfy the retailer's demand.

The retailer's order strategies are set as follows. (1) When the retailer's inventory level $x_{2}(k)<0$, the retailer asks for help from other SC. (2) When $x_{2}(k) \in\left[0, I_{0 r}\right]\left(I_{0 r}\right.$ is the retailer's expected inventory), the retailer normally orders the products from the manufacturer.

As shown in Figure 2, the fuzzy partitions of $x_{1}(k)$ and $x_{2}(k)$ are $H_{1}^{t}\left(x_{1}(k)\right)(t=1,2)$ and $H_{2}^{s}\left(x_{2}(k)\right)(s=1,2)$, respectively, and meet the conditions of SFP. Set $M_{1}^{1}=M_{1}^{2}=$ $H_{1}^{1}, M_{1}^{3}=M_{1}^{4}=H_{1}^{2}, M_{2}^{1}=M_{2}^{3}=H_{2}^{1}$, and $M_{2}^{2}=M_{2}^{4}=H_{2}^{2}$. In Figure $2, I_{0 \mathrm{~m}}=0.1\left(\times 10^{6}\right.$ sets $), I_{0 r}=0.8\left(\times 10^{5}\right.$ sets $)$, and $I_{m \max }$ and $I_{r \max }$ denote the manufacturer's maximal inventory level and the retailer's maximal inventory level, respectively.

In Figure 2, there is one MORG named $S$ that includes 4 rules in this system.

We can obtain this SC system with the production lead time and the ordering lead time as follows.

$$
\begin{aligned}
& R_{i} \text { : if } x_{1}(k) \text { is } M_{1}^{i} \text { and } x_{2}(k) \text { is } M_{2}^{i} \text {, then } \\
& \mathbf{x}(k+1)=\sum_{i=1}^{r} h_{i}\left[\mathbf{A}_{i} \mathbf{x}(k)+\mathbf{B}_{i} \mathbf{u}(k)+\mathbf{B}_{i 1} \mathbf{u}\left(k-\tau_{1}\right)\right. \\
& \left.+\mathbf{B}_{i 2} \mathbf{u}\left(k-\tau_{2}\right)+\mathbf{B}_{w i} \mathbf{w}(k)\right], \\
& \mathbf{z}(k)=\sum_{i=1}^{r} h_{i}\left[\mathbf{C}_{i} \mathbf{x}(k)+\mathbf{D}_{i} \mathbf{u}(k)+\mathbf{D}_{i 1} \mathbf{u}\left(k-\tau_{1}\right)\right. \\
& \left.+\mathbf{D}_{i 2} \mathbf{u}\left(k-\tau_{2}\right)\right]
\end{aligned}
$$

where $r=4, \mathbf{x}(k)=\left[\begin{array}{ll}x_{1}(k) & x_{2}(k)\end{array}\right]^{T}, \mathbf{u}(k)=\left[\begin{array}{ll}u_{1}(k) & u_{2}(k)\end{array}\right]^{T}$, $\tau_{1}$ is the production lead time, $\tau_{2}$ is the ordering lead time, $\mathbf{w}(k)=\left[\begin{array}{ll}0 & d(k)\end{array}\right]^{T}, d(k)$ denotes the customers' demands, and the system parameters are set as follows: $\mathbf{A}_{1}=\mathbf{0}, \mathbf{A}_{2}=\left[\begin{array}{ll}0 & 0 \\ 0 & 1\end{array}\right]$, $\mathbf{A}_{3}=\left[\begin{array}{ll}1 & 0 \\ 0 & 0\end{array}\right], \mathbf{A}_{4}=\left[\begin{array}{ll}1 & 0 \\ 0 & 1\end{array}\right], \mathbf{B}_{1}=\mathbf{B}_{3}=\left[\begin{array}{ll}1 & -1 \\ 0 & 1+\lambda\end{array}\right], \mathbf{B}_{2}=\mathbf{B}_{4}=$ $\left[\begin{array}{cc}1 & -1 \\ 0 & 1\end{array}\right], \mathbf{B}_{11}=\mathbf{B}_{21}=\mathbf{B}_{31}=\mathbf{B}_{41}=\left[\begin{array}{ll}1 & 0 \\ 0 & 0\end{array}\right], \mathbf{B}_{12}=\mathbf{B}_{22}=$ $\mathbf{B}_{32}=\mathbf{B}_{42}=\left[\begin{array}{ll}0 & 0 \\ 0 & 1\end{array}\right], \mathbf{B}_{w 1}=\mathbf{B}_{w 2}=\mathbf{B}_{w 3}=\mathbf{B}_{w 4}=\left[\begin{array}{cc}0 & 0 \\ 0 & -1\end{array}\right]$, $\mathbf{C}_{1}=\mathbf{0}, \mathbf{C}_{2}=\left[\begin{array}{ll}0 & c_{r 2}\end{array}\right], \mathbf{C}_{3}=\left[\begin{array}{ll}c_{r 1} & 0\end{array}\right], \mathbf{C}_{4}=\left[\begin{array}{ll}c_{r 1} & c_{r 2}\end{array}\right]$, $\mathbf{D}_{1}=\left[\begin{array}{ll}c_{m J} & c_{0}+c_{0 L}\end{array}\right], \mathbf{D}_{2}=\left[\begin{array}{ll}c_{m J} & 0\end{array}\right], \mathbf{D}_{3}=\left[\begin{array}{ll}c_{m} & c_{0}+c_{0 L}\end{array}\right]$, $\mathbf{D}_{4}=\left[\begin{array}{ll}c_{m} & c_{0}\end{array}\right], \mathbf{D}_{11}=\mathbf{D}_{21}=\left[\begin{array}{ll}c_{m J} & 0\end{array}\right], \mathbf{D}_{31}=\mathbf{D}_{41}=\left[\begin{array}{ll}c_{m} & 0\end{array}\right]$, $\mathbf{D}_{12}=\mathbf{D}_{32}=\left[\begin{array}{ll}0 & c_{0}+c_{0 L}\end{array}\right], \mathbf{D}_{22}=\mathbf{D}_{42}=\left[\begin{array}{ll}0 & c_{0}\end{array}\right]$, where $c_{r 1}$ and $c_{r 2}$ are the manufacturer's unit inventory cost and the retailer's unit inventory cost, respectively, $c_{r 1}=0.13\left(\times 10^{6} \mathrm{RMB}\right)$ and $c_{r 2}=0.30\left(\times 10^{6} \mathrm{RMB}\right), c_{m}$ is the unit manufacturing 


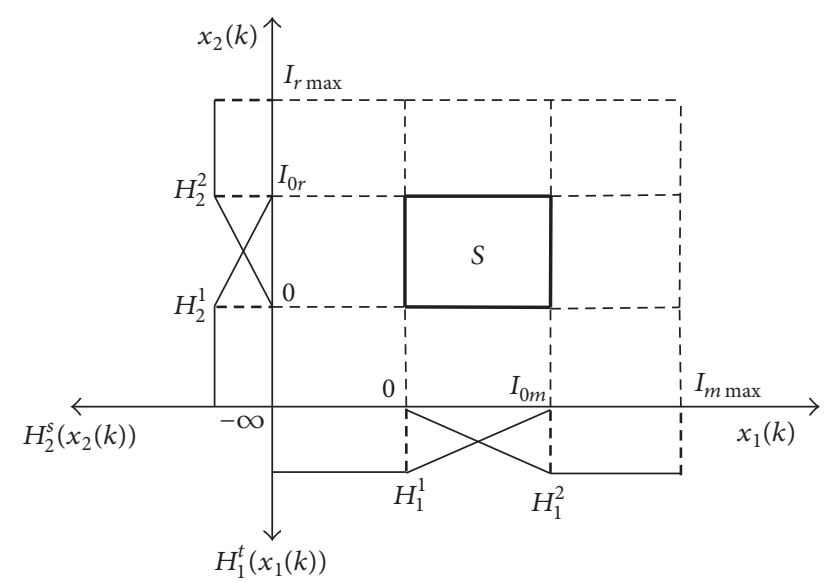

FIGURE 2: Fuzzy membership functions of inventory input variables.

cost when $x_{1}(k) \in\left[0, D_{0 m}\right], c_{m}=1.06\left(\times 10^{6} \mathrm{RMB}\right), c_{m J}$ is the unit manufacturing cost under JIT condition, $c_{m J}=$ $1.87\left(\times 10^{6} \mathrm{RMB}\right), c_{0}$ is the retailer's unit ordering cost when $x_{2}(k) \in\left[0, D_{0 r}\right], c_{0}=1.66\left(\times 10^{6} \mathrm{RMB}\right), c_{0 L}$ is the retailer's unit ordering cost from the manufacturers in the different SCs, $c_{0 L}=1.91\left(\times 10^{6} \mathrm{RMB}\right)$, and $\lambda$ is the supplying rate from other SCs, $0<\lambda \leq 1, \lambda=0.52$.

Then the fuzzy controller is designed as follows:

$$
\begin{array}{ll}
K^{i} \text { : If } \quad x_{1}(k) \text { is } M_{1}^{i}, x_{2}(k) \text { is } M_{2}^{i}, \\
\text { then } \quad \mathbf{u}(k)=-\sum_{i=1}^{r} h_{i} \mathbf{K}_{i 1} \mathbf{x}(k), \\
\mathbf{u}\left(k-\tau_{1}\right)=-\sum_{i=1}^{r} h_{i} \mathbf{K}_{i 11} \mathbf{x}\left(k-\tau_{1}\right), \\
\mathbf{u}\left(k-\tau_{2}\right)=-\sum_{i=1}^{r} h_{i} \mathbf{K}_{i 21} \mathbf{x}\left(k-\tau_{2}\right) .
\end{array}
$$

4.2. Simulation Analysis. When $\gamma=0.6$, this SC fuzzy system is robust stable by being found the following local matrices in MORG:

$$
\begin{aligned}
\mathbf{P}_{1} & =\left[\begin{array}{ll}
292.5161 & -55.5053 \\
-55.5053 & 119.3743
\end{array}\right], \\
\mathbf{Q}_{11} & =\mathbf{Q}_{21}=\left[\begin{array}{cc}
55.8514 & -17.0739 \\
-17.0739 & 11.1860
\end{array}\right] .
\end{aligned}
$$

In verifying the advantages of the proposed fuzzy robust $H_{\infty}$ control strategy for this nonlinear SC fuzzy system, the simulation experiments will be performed.

The initial values of states are $x_{1}(0)=0.9\left(\times 10^{5}\right.$ sets $)$, $x_{2}(0)=0.1\left(\times 10^{5}\right.$ sets $)$, and the normal values are set as $\vec{x}_{1}(k)=1.5$ ( $\times 10^{6}$ sets), $\vec{x}_{2}(k)=0.5\left(\times 10^{5}\right.$ sets $), \vec{u}_{1}(k)=$ $0.1\left(\times 10^{6}\right.$ sets $), \vec{u}_{2}(k)=0.6\left(\times 10^{5}\right.$ sets $), \vec{z}(k)=0.5\left(\times 10^{6}\right.$ $\mathrm{RMB}$ ), the production lead time $\tau_{1}=2$ (in weeks) and the

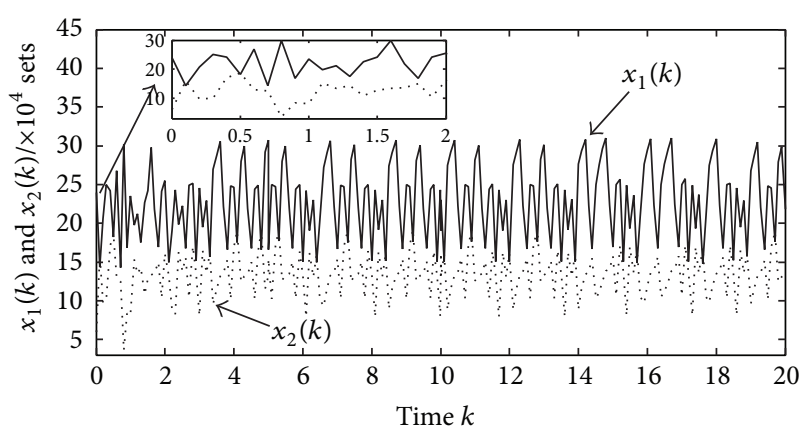

FIGURE 3: Evolution processes of inventory levels under the robust $H_{\infty}$ control strategy.

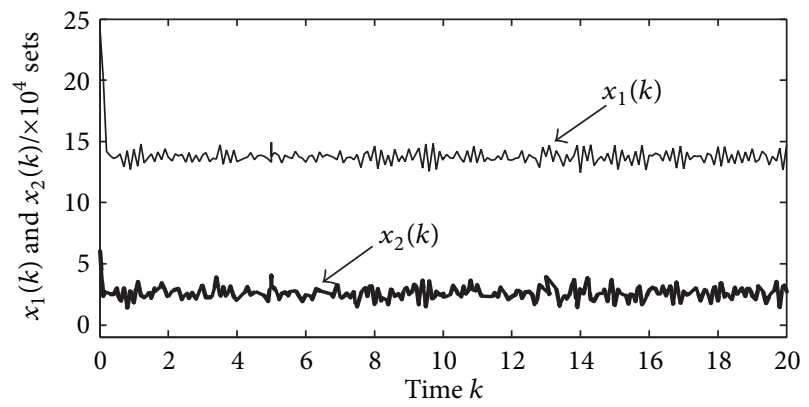

FIGURE 4: Evolution processes of inventory level under the fuzzy robust $H_{\infty}$ control strategy.

ordering lead time $\tau_{2}=2$ (in weeks), and the customers' demands $d(k) \sim N\left(3,0.5^{2}\right)$. The simulation results are expressed as the actual values.

To show the superiority of our proposed control strategy, the robust $H_{\infty}$ control strategy and our control strategy are provided for comparison. The robust $H_{\infty}$ control strategy can be described as follows:

$$
\begin{array}{ll}
K^{i}: \text { If } & x_{1}(k) \text { is } M_{1}^{i}, x_{2}(k) \text { is } M_{2}^{i}, \\
\text { then } & \mathbf{u}(k)=-\mathbf{K}_{i 1} \mathbf{x}(k), \\
& \mathbf{u}\left(k-\tau_{1}\right)=-\mathbf{K}_{i 11} \mathbf{x}\left(k-\tau_{1}\right), \\
& \mathbf{u}\left(k-\tau_{2}\right)=-\mathbf{K}_{i 21} \mathbf{x}\left(k-\tau_{2}\right) .
\end{array}
$$

Figures 3, 5, and 7 illustrate the evolution processes of inventory levels, production and ordering quantity, and total cost under the robust $H_{\infty}$ control strategy.

By using our proposed control strategy, that is to say, fuzzy robust $H_{\infty}$ control strategy, the simulation results are shown in Figures 4, 6, and 8.

Figures 3 and 4 are selected as an example to analyze the control effects of two control approaches. As shown in Figures 3 and 4 , the differences of the crests and the troughs for the manufacturer's inventory level $x_{1}(k)$ under the robust $H_{\infty}$ control strategy and the fuzzy robust $H_{\infty}$ control strategy are $30 \times 10^{4}$ sets-15 $\times 10^{4}$ sets $=15 \times 10^{4}$ sets and $15 \times 10^{4}$ sets$12 \times 10^{4}$ sets $=2 \times 10^{4}$ sets, respectively. 


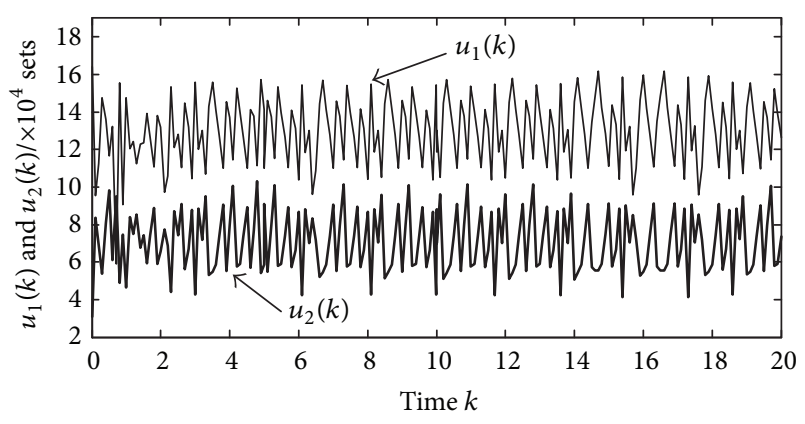

FIGURE 5: Evolution processes of production and ordering quantity under the robust $H_{\infty}$ control strategy.

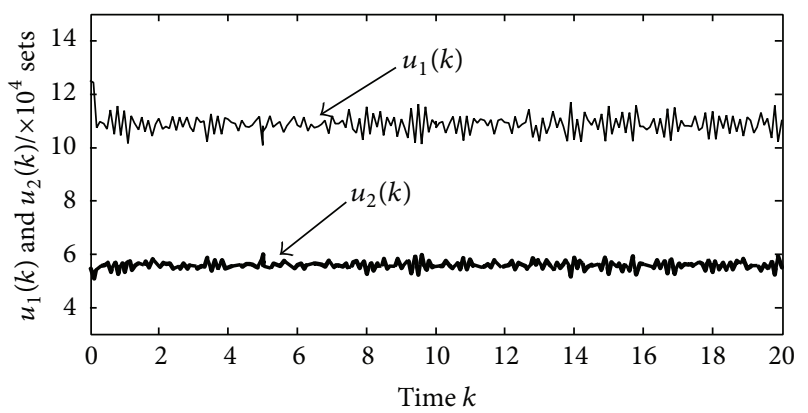

FIGURE 6: Evolution processes of production and ordering quantity under the fuzzy robust $H_{\infty}$ control strategy.

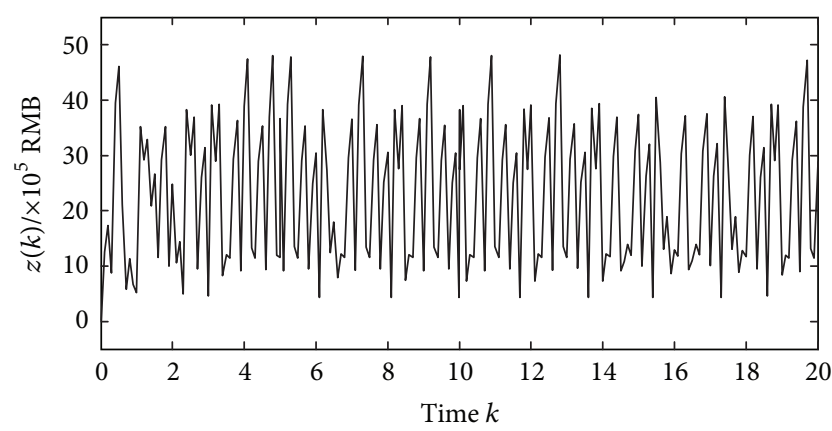

FIGURE 7: Evolution process of total cost under the robust $H_{\infty}$ control strategy.

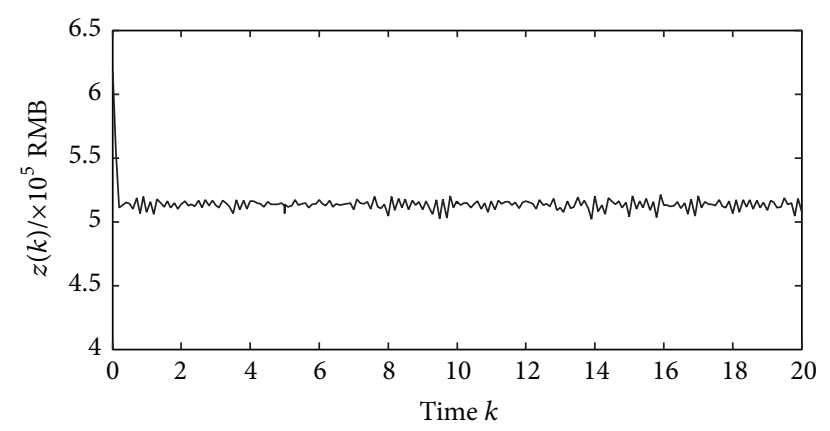

FIGURE 8: Evolution process of total cost under the fuzzy robust $H_{\infty}$ control strategy.

\section{Conclusion}

The robust stabilization problem of the operational process of the dynamic SC system with lead times has been studied. Utilizing T-S fuzzy system, we establish the nonlinear dynamic SC fuzzy system. To restrain the disturbances of lead times, switching actions among subsystems, and customers' stochastic demands, a new fuzzy robust $H_{\infty}$ control strategy has been proposed by utilizing the definition of MORG. In addition, this strategy can guarantee that the nonlinear SC system with lead times is robustly asymptotically stable. In the simulation research, we have discussed the operational process of two-stage nonlinear SC system with the production lead time and the ordering lead time, and the simulation tests have verified the advantage of the proposed fuzzy robust control strategy. Our designed strategy can be applied to some node companies in supply chain system.

\section{Conflicts of Interest}

The authors declare that they have no conflicts of interest.

\section{Acknowledgments}

This work was supported by the National Social Science Foundation of China (17BGL222).

\section{References}

[1] K. Zimmer, "Supply chain coordination with uncertain just-intime delivery," International Journal of Production Economics, vol. 77, no. 1, pp. 1-15, 2002.

[2] G. Zhang, J. Shang, and W. Li, "Collaborative production planning of supply chain under price and demand uncertainty," European Journal of Operational Research, vol. 215, no. 3, pp. 590-603, 2011.

[3] S. Park, T.-E. Lee, and C. S. Sung, "A three-level supply chain network design model with risk-pooling and lead times," Transportation Research Part E: Logistics and Transportation Review, vol. 46, no. 5, pp. 563-581, 2010.

[4] R. J. Tersine and E. A. Hummingbird, "Lead-time reduction: the search for competitive advantage," International Journal of Operations and Production Management, vol. 15, no. 2, pp. 8-18, 1995.

[5] S. Mahajan and V. Venugopal, "Value of information sharing and lead time reduction in a supply chain with autocorrelated demand," Technology Operation Management, vol. 2, no. 1, pp. 39-49, 2011.

[6] M. Leng and M. Parlar, "Lead-time reduction in a two-level supply chain: non-cooperative equilibria vs. coordination with a profit-sharing contract," International Journal of Production Economics, vol. 118, no. 2, pp. 521-544, 2009.

[7] Y. Li, X. J. Xu, and F. Ye, "Supply chain coordination model with controllable lead time and service level constraint," Computers Industrial Engineering, vol. 61, no. 3, pp. 858-864, 2011.

[8] C. H. Glock, "Lead time reduction strategies in a singlevendorsingle-buyer integrated inventory model with lot sizedependent lead times and stochastic demand," International Journal of Production Economics, vol. 136, no. 1, pp. 37-44, 2012. 
[9] J. K. Jha and K. Shanker, "An integrated inventory problem with transportation in a divergent supply chain under service level constraint," Journal of Manufacturing Systems, vol. 33, no. 4, pp. 462-475, 2014.

[10] J. Heydari, "Lead time variation control using reliable shipment equipment: An incentive scheme for supply chain coordination," Transportation Research Part E: Logistics and Transportation Review, vol. 63, pp. 44-58, 2014.

[11] C. A. Garcia, A. Ibeas, J. Herrera, and R. Vilanova, "Inventory control for the supply chain: An adaptive control approach based on the identification of the lead-time," Omega, vol. 40, no. 3, pp. 314-327, 2012.

[12] $\mathrm{H}$. $\mathrm{Xu}$ and $\mathrm{G}$. Rong, "A minimum variance control theory perspective on supply chain lead time uncertainty," Industrial and Engineering Chemistry Research, vol. 51, no. 27, pp. 92759286, 2012.

[13] A. A. Taleizadeh, S. T. A. Niaki, N. Shafii, R. G. Meibodi, and A. Jabbarzadeh, "A particle swarm optimization approach for constraint joint single buyer-single vendor inventory problem with changeable lead time and (r,Q) policy in supply chain," International Journal of Advanced Manufacturing Technology, vol. 51, no. 9-12, pp. 1209-1223, 2010.

[14] C. Li and S. Liu, "A robust optimization approach to reduce the bullwhip effect of supply chains with vendor order placement lead time delays in an uncertain environment," Applied Mathematical Modelling. Simulation and Computation for Engineering and Environmental Systems, vol. 37, no. 3, pp. 707-718, 2013.

[15] C. A. Garcia, A. Ibeas, R. Vilanova, and J. Herrera, "Leadtime identification for inventory control of the supply chain," in Proceedings of the 20th Mediterranean Conference on Control \& Automation, pp. 728-733, Barcelona, Spain, July 2012.

[16] X.-J. Han, A.-M. Feng, and B.-L. Zhang, "Approximate optimal inventory control of supply chain networks with lead time," in Proceedings of the 27th Chinese Control and Decision Conference, CCDC 2015, pp. 4523-4528, May 2015.

[17] K. K. Movahed and Z.-H. Zhang, "Robust design of (s,S) inventory policy parameters in supply chains with demand and lead time uncertainties," International Journal of Systems Science. Principles and Applications of Systems and Integration, vol. 46, no. 12, pp. 2258-2268, 2015.

[18] X. Wang and S. M. Disney, "Mitigating variance amplification under stochastic lead-time: the proportional control approach," European Journal of Operational Research, vol. 256, no. 1, pp. 151162, 2017.

[19] H. J. Lee, J. B. Park, and G. Chen, "Robust fuzzy control of nonlinear systems with parametric uncertainties," IEEE Transactions on Fuzzy Systems, vol. 9, no. 2, pp. 369-379, 2001.

[20] H. Yang and J. Zhao, "Robust control for a class of uncertain switched fuzzy systems," Journal of Control Theory and Applications, vol. 5, no. 2, pp. 184-188, 2007.

[21] Y. Cui, K. Liu, Y. Zhao, and X. Wang, "Robust Ho control for a class of uncertain switched fuzzy time-delay systems based on T-S models," Mathematical Problems in Engineering, vol. 2013, Article ID 234612, 2013.

[22] L. Teng, Y. Wang, W. Cai, and H. Li, "Robust model predictive control of discrete nonlinear systems with time delays and disturbances via T-S fuzzy approach," Journal of Process Control, vol. 53, pp. 70-79, 2017.

[23] Qi. Ruiyun, T. Gang, B. Jiang, and C. Tan, "Adaptive control schemes for discrete-time T-S fuzzy systems with unknown parameters and actuator failures," in Proceedings of the 2011
American Control Conference, pp. 3748-3753, San Francisco, CA, USA, June 2011.

[24] C. M. Li and X. M. Tian, "Control based on LMIs for a class of time-delay switched system," Journal of Measurement Science \& Instrumentation, vol. 1, no. 3, pp. 293-296, 2010.

[25] X. D. Liu and Q. L. Zhang, "Approaches to quadratic stability conditions and Ho control designs for T-S fuzzy systems," IEEE Transactions on Fuzzy Systems, vol. 11, no. 6, pp. 830-839, 2003.

[26] Z.-H. Xiu and G. Ren, "Stability analysis and systematic design of Takagi-Sugeno fuzzy control systems," Fuzzy Sets and Systems. An International Journal in Information Science and Engineering, vol. 151, no. 1, pp. 119-138, 2005.

[27] X. P. Guan and C. L. Chen, "Delay-dependent guaranteed cost control for T-S fuzzy systems with time delays," IEEE Transactions on Fuzzy Systems, vol. 12, no. 2, pp. 236-249, 2004.

[28] S. Zhang, Y. Hou, and X. Zhao, "Robust stabilization for discrete uncertain Takagi-Sugeno fuzzy systems based on a piecewise Lyapunov function," Industrial and Engineering Chemistry Research, vol. 53, no. 17, pp. 7132-7140, 2014.

[29] S. Zhang, X. Li, and C. Zhang, "A fuzzy control model for restraint of bullwhip effect in uncertain closed-loop supply chain with hybrid recycling channels," IEEE Transactions on Fuzzy Systems, vol. 25, no. 2, pp. 475-482, 2017.

[30] B. Zhang and S. Xu, "Delay-Dependent Robust Ho control for uncertain discrete-time fuzzy systems with time-varying delays," IEEE Transactions on Fuzzy Systems, vol. 17, no. 4, pp. 809-823, 2009. 


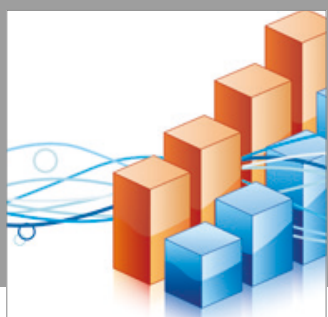

Advances in

Operations Research

vatersals

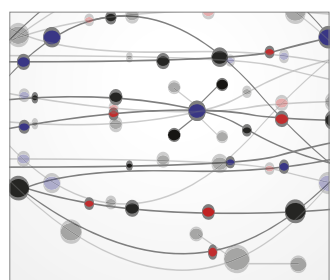

\section{The Scientific} World Journal
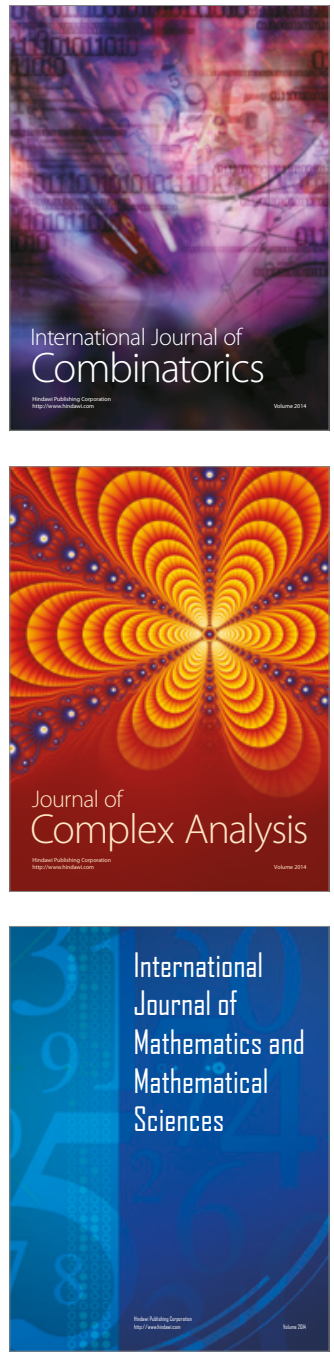
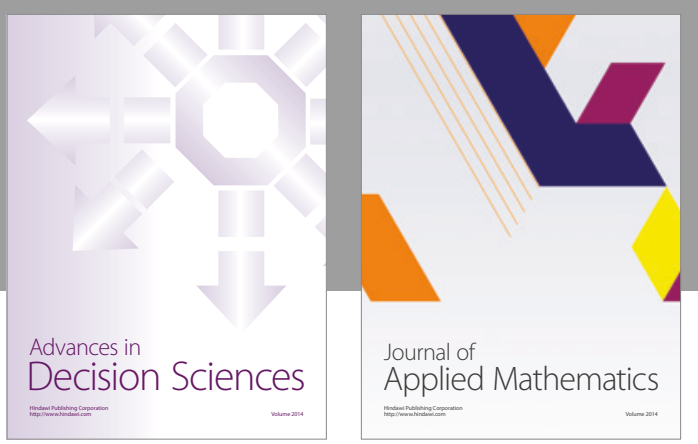

Algebra

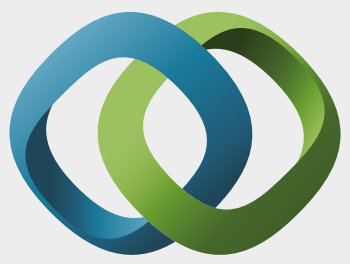

\section{Hindawi}

Submit your manuscripts at

https://www.hindawi.com
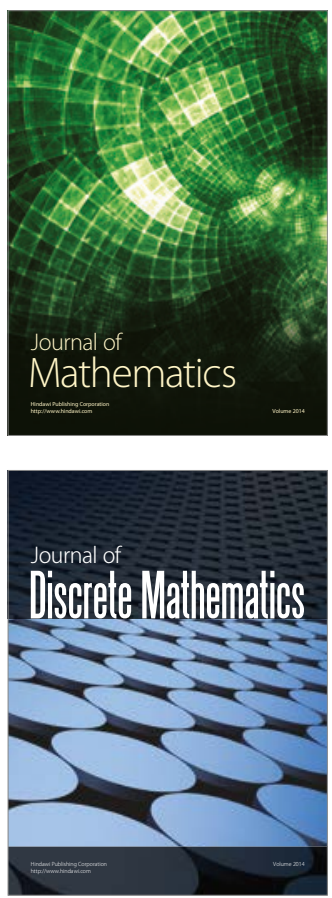

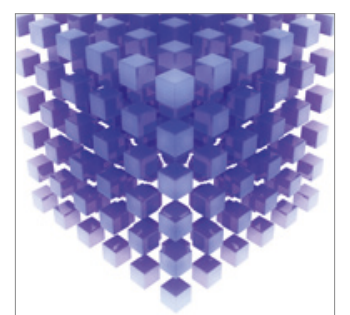

Mathematical Problems in Engineering
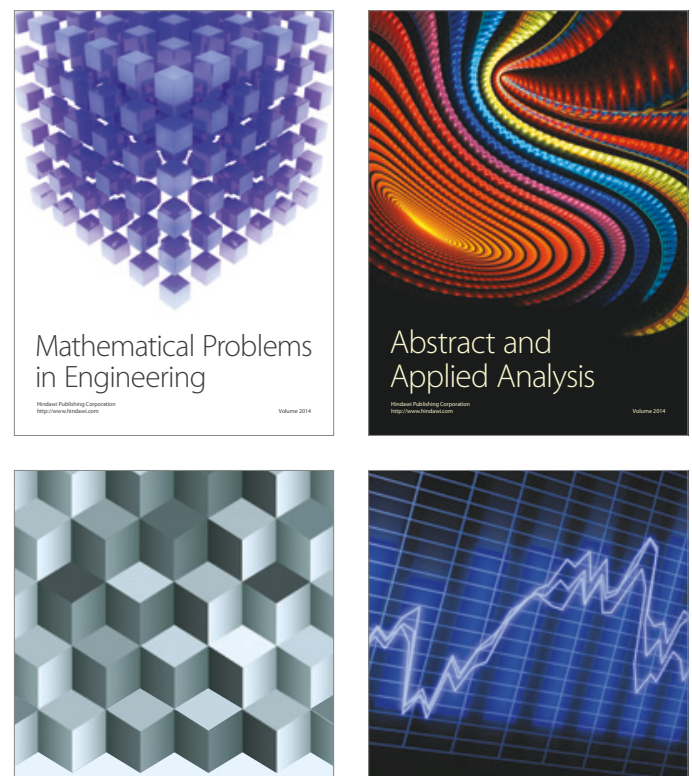

Journal of

Function Spaces

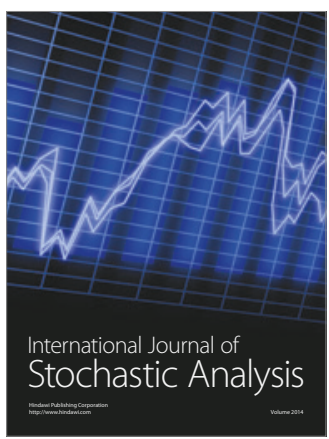

Probability and Statistics
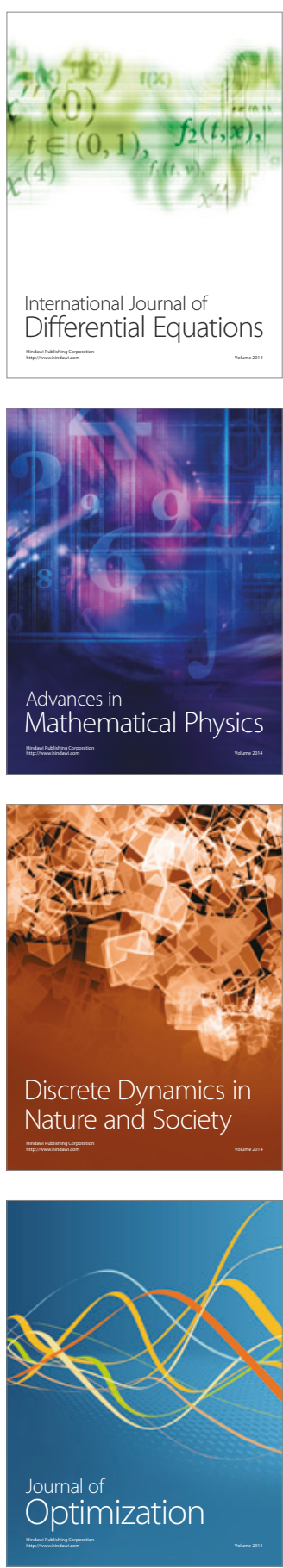